\title{
Treatment of stage III non-small cell lung cancer in the era of immunotherapy: pathological complete response to neoadjuvant pembrolizumab and chemotherapy
}

\author{
Zhang-Ru Yang ${ }^{1}$, Mi-Na Liu ${ }^{1}$, Jia-Hua Yu ${ }^{1}$, Yun-Hai Yang ${ }^{2}$, Tian-Xiang Chen ${ }^{2}$, Yu-Chen Han ${ }^{3}$, Lei Zhu ${ }^{3}$, \\ Ji-Kai Zhao ${ }^{3}$, Xiao-Long Fu ${ }^{1}$, Xu-Wei Cai ${ }^{1}$ \\ ${ }^{1}$ Department of Radiation Oncology, ${ }^{2}$ Shanghai Lung Cancer Center, ${ }^{3}$ Department of Pathology, Shanghai Chest Hospital, Shanghai Jiao Tong \\ University, Shanghai, China \\ Contributions: (I) Conception and design: All authors; (II) Administrative support: None; (III) Collection and assembly of data: ZR Yang, MN Liu, YC \\ Han, XW Cai, XL Fu; (IV) Data analysis and interpretation: All authors; (V) Manuscript writing: All authors; (VI) Final approval of manuscript: All \\ authors. \\ Correspondence to: Xu-Wei Cai. Department of Radiation Oncology, Shanghai Chest Hospital, Shanghai Jiao Tong University, No. 241 West Huaihai \\ Road, Shanghai 200030, China. Email: birdhome2000@163.com.
}

\begin{abstract}
Non-small cell lung cancer (NSCLC) accounts for about $85 \%$ of all lung cancers. The expected 5 -year survival of stage III NSCLC ranges from $13 \%$ to $36 \%$ for stage III. Due to the heterogeneity and poor efficacy of stage III patients, there is great controversy on how to optimize the therapy strategy. Immunotherapy is providing better clinical efficacy to more NSCLC patients, and is rapidly extending its range of care from advanced stage to locally advanced stage and early stage NSCLC. Due to the patient's strong treatment intention, drug availability, and a few encouraging results from clinical trials (NADIM, NCT02716038, etc.), the authors observed a case of stage III NSCLC that achieved complete remission after receiving neoadjuvant chemotherapy combined with immunotherapy. In view of such a satisfactory result in neoadjuvant therapy, this article discusses how comprehensive treatment for stage III NSCLC patients may be conducted and the manner in which various therapeutic techniques can be mastered in the era of immunotherapy. Immunotherapy has opened the exploratory space for finding resolutions to numerous challenges of treating stage III NSCLC. Further clinical studies and exploration of personalized treatment, guided by imaging data, and clinical and pathological biomarkers are imperative for the benefit of these patients.
\end{abstract}

Keywords: Non-small cell lung cancer (NSCLC); neoadjuvant treatment; programmed death receptor-1 (PD-1); chemotherapy

Submitted May 26, 2020. Accepted for publication Sep 24, 2020.

doi: $10.21037 /$ tlcr-20-896

View this article at: http://dx.doi.org/10.21037/tlcr-20-896

\section{Introduction}

Non-small cell lung cancer (NSCLC) accounts for about $85 \%$ of all lung cancers (1). NSCLC is related to environmental factors and long-term genetic interactions. Those related factors include individual's own factors, age, heredity, immune system and nutritional status. And the environmental factors include smoking, occupational factors, environmental pollution, etc. Among NSLC cases, $30 \%$ are patients with stage III NSCLC who have larger size or metastatic lymph nodes in the mediastinum. The expected 5-year survival for stage III patients ranges from

\footnotetext{
$\wedge$ ORCID: 0000-0001-5579-0159.
} 
$13 \%$ to $36 \%$ (IIIA $36 \%$; IIIB $26 \%$; IIIC $13 \%$ ) (2). The survival rate garnered from perioperative platinum-based chemotherapy is only $5.4 \%$ higher than that provided by surgery alone, with rates of toxic effects of grade 3 or higher occurring in more than $60 \%$ of cases $(3,4)$. Consequently, more effective agents are urgently required to improve the effect of neoadjuvant therapy. In terms of the location of the tumor, the tumor of stage III NSCLC is still confined to the thoracic cavity and belongs to local growth. Compared with stage IV (also known as advanced stage) that has already occurred distant metastasis, stage III NSCLC is more suitable for immunotherapy.

Over the last few decades, instances of substantive progress in extending the survival of patients with stage III NSCLC have been rare, despite numerous clinical trials being conducted using cytotoxic chemotherapeutic agents and radiotherapy (5). Thus, a safe and effective treatment strategy for improving the outcomes of these patients requires more involved investigation.

Antibodies that block the immune inhibitory pathway of the programmed death 1 (PD-1) protein have provided a novel treatment advance in patients with NSCLC. These antibodies confer antitumor immunity, resulting in tumor regression and improved survival (6-10). PD-1/programmed death-ligand 1 (PD-L1) immunosuppressants are currently an area of intense NSCLC research, and have demonstrated significant value in treating advanced patients. For stage III inoperable patients, consolidation therapy after concurrent radiotherapy has achieved significant improvement in efficacy. However, how immunotherapy should be implemented in patients with stage III NSCLC who are operable or critically operable is still unclear.

The neoadjuvant immunotherapy trial results for stage I-IIIA NSCLC are summarized in Table 1. The results of these preliminary phase I and II studies suggest that immune checkpoint inhibitors (ICIs) are feasible and well tolerated in early NSCLC neoadjuvant therapy patients (11). Furthermore, pembrolizumab, the first Food and Drug Administration (FDA)-approved PD-1 inhibitor, has been shown to significantly improved outcomes in patients with advanced NSCLC (8,12-16).

The following is a report of one patient with stage III NSCLC. Due to the patient's strong treatment intention, drug availability, and a few encouraging results from clinical trials (NADIM, NCT02716038, etc.) a neoadjuvant treatment of chemotherapy plus pembrolizumab was administered and achieved an immediate and satisfactory effect. The details of treatment and efficacy are described below.

\section{Report of pathologic complete response (PCR) to neoadjuvant pembrolizumab and chemotherapy}

\section{Patient and treatment}

The patient was a male (54 years; height: $176 \mathrm{~cm}$; body weight: $72 \mathrm{~kg}$ ) who was diagnosed with squamous cell carcinoma (SCC) in the lower lobe orifice of the right lung. The primary lesion was about $3.5 \mathrm{~cm}$ in diameter and, according to the positron emission tomography (PET) scan, had formed a single mass, with right hilar and mediastinal (4R) lymphadenopathy.

The study was conducted in accordance with the Declaration of Helsinki (as revised in 2013). Written informed consent was obtained from the patient for publication of this manuscript and any accompanying images.

Performance status (PS): Moderate. Histopathology: SSC, carcinoembryonic antigen (CEA): normal. Diagnostic: NSCLC. Immunochemistry detection showed that PD-L1 expression was $5 \%$ in of biopsied tumor cells tissues of the primary lung tumor. Staging of the patient was c-T2aN2M0. Surgical treatment (right lower lobectomy plus lymphadenectomy) was not possible due to the tumor mass' close proximity to right the right main bronchus.

Treatment (Figure 1) was neoadjuvant therapy with pembrolizumab and chemotherapy (compassionate use) with surgery and adjuvant therapy. The treatment regimen (about 2.5 months) proceeded as follows: (I) administration of 2 cycles of pembrolizumab $200 \mathrm{mg}+\mathrm{TP}$ regimen (paclitaxel liposome combined with platinum, days 1 and 22); (II) Right lower lobectomy plus lymph node dissection were performed 3 weeks later; (III) adjuvant therapy with pembrolizumab and TP regimen administered 2 months after surgery. Thus, 7 days after diagnosis, pembrolizumab and chemotherapy were administered simultaneously.

For the clinical course (Figure 2), about 3 weeks after 2 cycles of neoadjuvant therapy (Table 2; Figures 1C,2B), no side effects of pembrolizumab were observed. Chest computed tomography (CT) showed a decline of the lung mass (initial diameter, $3.5 \mathrm{~cm}$; 3 -week diameter, $1.5 \mathrm{~cm}$ ). The right hilar and mediastinal lymphadenopathy showed a decrease compared to the initial diameter.

According to the CT scan, the primary tumor lesion showed a partial response (PR), and two new nodules were present in the right upper lobe, and were considered likely 
Table 1 The neoadjuvant immunotherapy trial results for stage I-IIIA non-small cell lung cancer (NSCLC)

\begin{tabular}{|c|c|c|c|c|c|c|}
\hline Trial & NCT & Phase & $\begin{array}{l}\text { Stage of } \\
\text { disease }\end{array}$ & Cases & Intervention regimen & Results \\
\hline $\begin{array}{l}\text { Immune activation } \\
\text { in early-stage } \\
\text { NSCLC patients } \\
\text { receiving neoadjuvant } \\
\text { chemotherapy plus } \\
\text { ipilimumab }\end{array}$ & NCT01820754 & I & $\begin{array}{l}\text { IIA } 3 \text { cases } \\
\text { IIB } 2 \text { cases } \\
\text { IIIA } 19 \text { cases }\end{array}$ & 24 & $\begin{array}{l}\text { Neoadjuvant: paclitaxel \& } \\
\text { carboplatin + ipilimumab, } \\
\text { two cycles before surgery, } \\
\text { Q3W }\end{array}$ & $\begin{array}{l}\text { Disease control rate: } 91 \% \\
\text { Surgical completion rate: } 13 / 24 \\
\geq \text { grade } 3 \text { adverse event rate: } 46 \%\end{array}$ \\
\hline $\begin{array}{l}\text { Neoadjuvant } \\
\text { programmed death } \\
\text { receptor-1 (PD-1) } \\
\text { blockade in } \\
\text { resectable lung } \\
\text { cancer }\end{array}$ & NCT02259621 & ॥ & $\begin{array}{l}\text { I } 4 \text { cases } \\
\text { II } 10 \text { cases } \\
\text { IIIA } 7 \text { cases }\end{array}$ & 21 & $\begin{array}{l}\text { Neoadjuvant: nivolumab } 3 \\
\text { mg/kg, two cycles before } \\
\text { surgery, Q2W }\end{array}$ & $\begin{array}{l}\text { Disease control rate: } 96 \% \\
\text { Major pathological response } \\
\text { (MPR): } 45 \% \\
\text { Pathological complete response } \\
\text { (pCR): } 15 \% \\
\text { Surgical completion rate: } 20 / 21 \\
\geq \text { grade } 3 \text { adverse event rate: } 5 \%\end{array}$ \\
\hline $\begin{array}{l}\text { Neoadjuvant } \\
\text { atezolizumab }+ \\
\text { chemotherapy } \\
\text { in patients with } \\
\text { resectable NSCLC }\end{array}$ & NCT02716038 & II & $\begin{array}{l}\text { IIA } 2 \text { cases } \\
\text { IIB } 1 \text { cases } \\
\text { IIIA } 11 \text { cases }\end{array}$ & 14 & $\begin{array}{l}\text { Neoadjuvant: } \\
\text { atezolizumab 1,200 mg + } \\
\text { carboplatin, Q3W; nab- } \\
\text { paclitaxel, D1, D8, D15 }\end{array}$ & $\begin{array}{l}\text { Disease control rate: } 100 \% \\
\text { MPR: } 50 \% \\
\text { Pathological complete response: } \\
21.4 \% \\
\text { Surgical completion rate: } 11 / 14 \\
\text { Chemotherapy dose adjustment: } \\
64.3 \%\end{array}$ \\
\hline
\end{tabular}

to be a pseudo progression.

Surgical treatment (right lower lobectomy plus lymphadenectomy) was performed after two cycles of neoadjuvant therapy.

The patient's pathology (Figure 3) was considered to be hyperplasia of interstitial fibrous tissue around the 


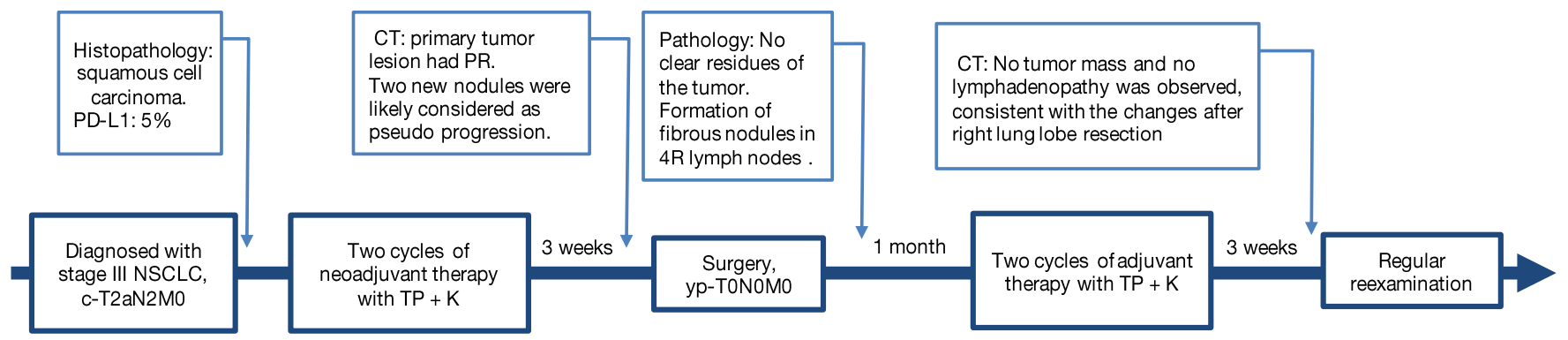

Figure 1 The treatment process for stage III non-small cell lung cancer.

dorsal bronchus of the lower lobe of the right lung, with infiltration of lymphocytes, thickening of small vessel walls, mucus degeneration, and retention of tissue cells, and mucus in the peripheral alveolar cavity. There were no clear residues of the tumor, which were considered to be changed after neoadjuvant immunotherapy. The size of the lesion was $1.5 \times 1 \times 0.8 \mathrm{~cm}^{3}$. No obvious lesions were found at the incision of the bronchus. No metastasis was found in six groups of lymph nodes. For group 4, the formation of fibrous nodules in the lymph nodes with histocellular response was observed. Pathological staging was ypTONOMO.

Response evaluation was performed 1 month after surgery (Table 2; Figures $1 E, 2 D)$ and 1 month after adjuvant therapy (Table 2; Figures 1G,2F).

The clinical stage of this case was c-T2aN2M0. After diagnosis, the patient was treated with two cycles of neoadjuvant therapy with pembrolizumab, combined with chemotherapy with a regimen of paclitaxel and cisplatin. Surgical intervention was administered to the right lower lobe, and hilar and mediastinal lymph nodes. Staging after surgery was yp-TONOM0. The patient was then treated with 2 cycles of adjuvant therapy with pembrolizumab plus TP regimen. Complete response (CR) was achieved after 2 cycles of neoadjuvant pembrolizumab plus chemotherapy. The preliminary results suggest that there is a good opportunity for chemotherapy plus immunotherapy. Thus, the therapeutic strategies and the application of treatment techniques for stage III NSCLC patients available in the current era of immunotherapy were considered.

\section{Is the patient achieving $p C R$ after neoadjuvant chemotherapy plus immunotherapy an isolated case or $s$ common phenomenon?}

Forde et al. were first to investigate neoadjuvant PD-1 blockade in resectable lung cancer, reporting that 9 of the 20 resected tumors (45\%) achieved major pathological response (MPR). Responses were observed in both PD-L1 positive and PD-L1 negative tumors. There was a significant correlation between pathological response and tumor mutation burden (TMB) before treatment. Furthermore, 8 of the 9 patients evaluated had a systematic increase in the number of $\mathrm{T}$ cell clones, which were found in both tumor and peripheral blood after PD-1 blockade.

In a multicenter phase II study of neoadjuvant chemotherapy, PR to neoadjuvant treatment was observed in 43 of 78 patients $(55 \%)$ (17). Chaft et al. reported a similar result with 20 of 45 patients achieving PR for an objective response rate (ORR) of $45 \%$ (18). However, almost no cases of CR were observed in stage III NSCLC patients who had undergone neoadjuvant chemotherapy. Several preoperative chemoradiotherapy studies assessed the pathologic response in locally advanced NSCLC cases and found pCR rates ranging from $17 \%$ to $45 \%$ (19-26).

In this case discussed here, CR was achieved after completion of two cycles of neoadjuvant pembrolizumab plus chemotherapy. Similar results from a phase II multicenter exploratory study (NADIM) showed that $83 \%$ achieved MPR, and $71 \%$ achieved pCR. Downstaging was seen in $90 \%$ of cases. According to response evaluation criteria in solid tumors (RECIST), 29 patients (71\%) achieved PR and 3 patients (7\%) achieved CR. A multicenter study (LCMC3) of neoadjuvant atezolizumab (atezo) in resectable NSCLC reported that 4 out of 82 patients had pCR (pCR: 5\%). According to RECIST, 6 patients were $\mathrm{PR}, 72$ were stable disease (SD), and 4 were progressive disease (PD). These results indicate that the overall efficacy of neoadjuvant immunotherapy seems to be satisfactory and that the addition of immunotherapy to chemotherapy-based neoadjuvant treatment may provide further benefits. 

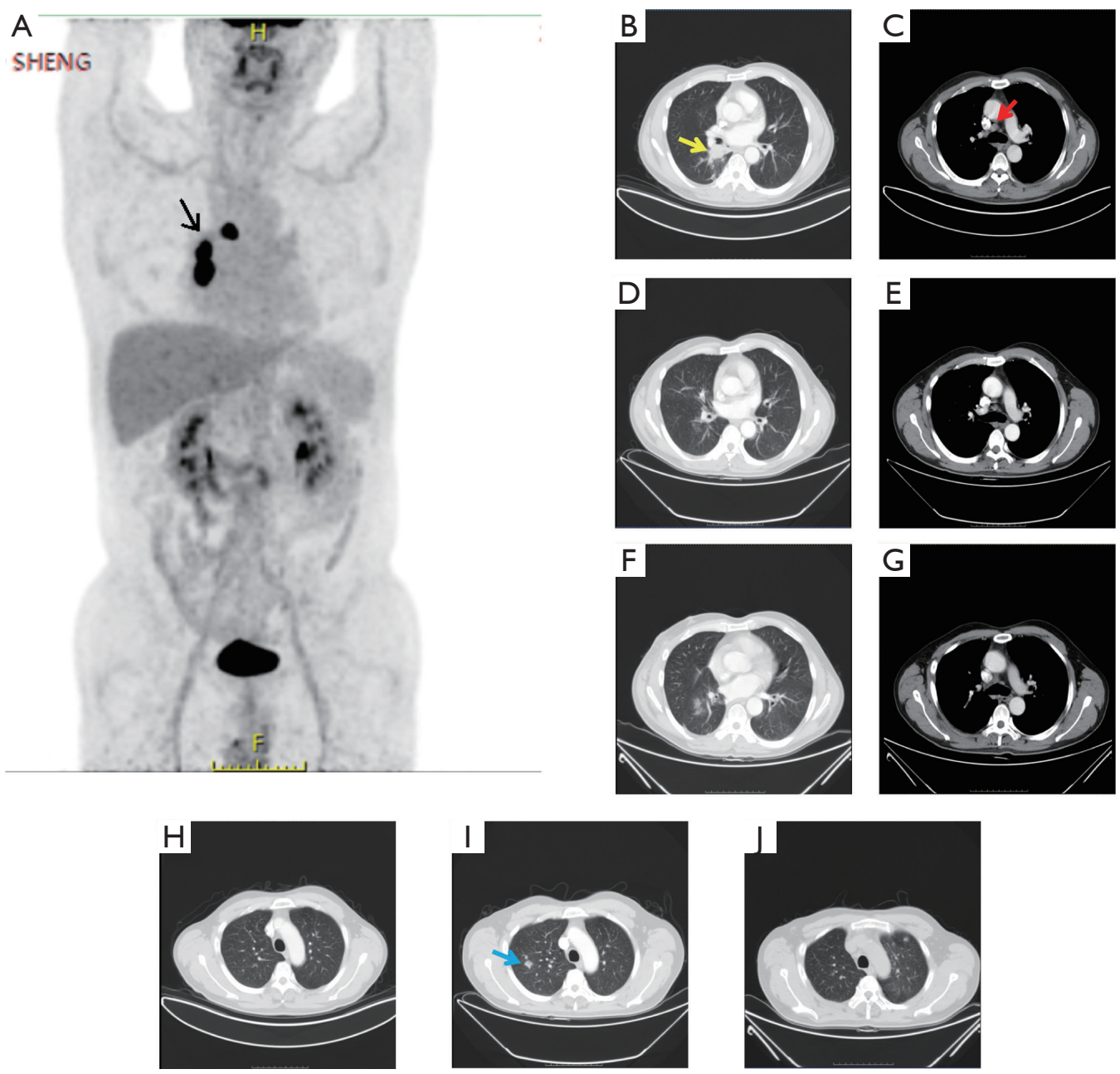

Figure 2 The clinical course of stage III NSCLC. (A) A whole body PET scan was performed when the patient was diagnosed with NSCLC. Intense FDG uptake was visualized in the lower lobe orifice of the right lung (diameter: $3.5 \mathrm{~cm}$; black arrow), and in the right hilar and mediastinal (4R) lymph nodes. In this image, the remainder of the FDG uptake was located in the excretory system. (B) Before treatment, a CT scan also showed that there was a mass in the right lung lower lobe orifice (diameter: $3.5 \mathrm{~cm}$; yellow arrow), (C) with right hilar and mediastinal (4R) lymphadenopathy (red arrow). (D) After two cycles of neoadjuvant pembrolizumab and chemotherapy, the tumors in the lung, and (E) the right hilar and mediastinal (4R) lymph nodes had nearly disappeared. (F) After surgery, a CT scan indicated no tumor mass, which was consistent with the changes after right lung lobe resection. (G) Hilar and mediastinal adenopathies were not observed. Right pleural effusion was observed. $(\mathrm{H})$ No nodules in the right upper lobe were found before neoadjuvant therapy. (I) After two cycles of neoadjuvant pembrolizumab and chemotherapy, a chest CT scan revealed two new nodules in the right upper lobe (blue arrow), which were considered likely to be pseudo progression. (J) Re-examination after surgery with CT revealed new nodules in the same location of the right upper lobe had disappeared. NSCLC, non-small cell lung cancer.

In this case, the pathological evidence supports the possibility that some NSCLC patients may obtain clinical benefit from immunotherapy plus chemotherapy, due to infiltration of immune-cell into the tumor. The NEOSTAR study also demonstrated that levels of CD3+, CD103+, and tissue resident memory $\mathrm{CD} 8+$ tumor-infiltrating lymphocytes (TILs) were higher in the nivolumab plus ipilimumab (NI-) regimen compared with $\mathrm{N}$-treated tumors. Meanwhile, it was observed in radiographic images that new nodules appeared briefly around the tumor lesion, which was considered pseudo progression. We thought this phenomenon was the result of immune-cell infiltration, 
Table 2 Patient evolution course: neoadjuvant therapy with pembrolizumab and chemotherapy (compassionate use) plus surgery plus adjuvant therapy

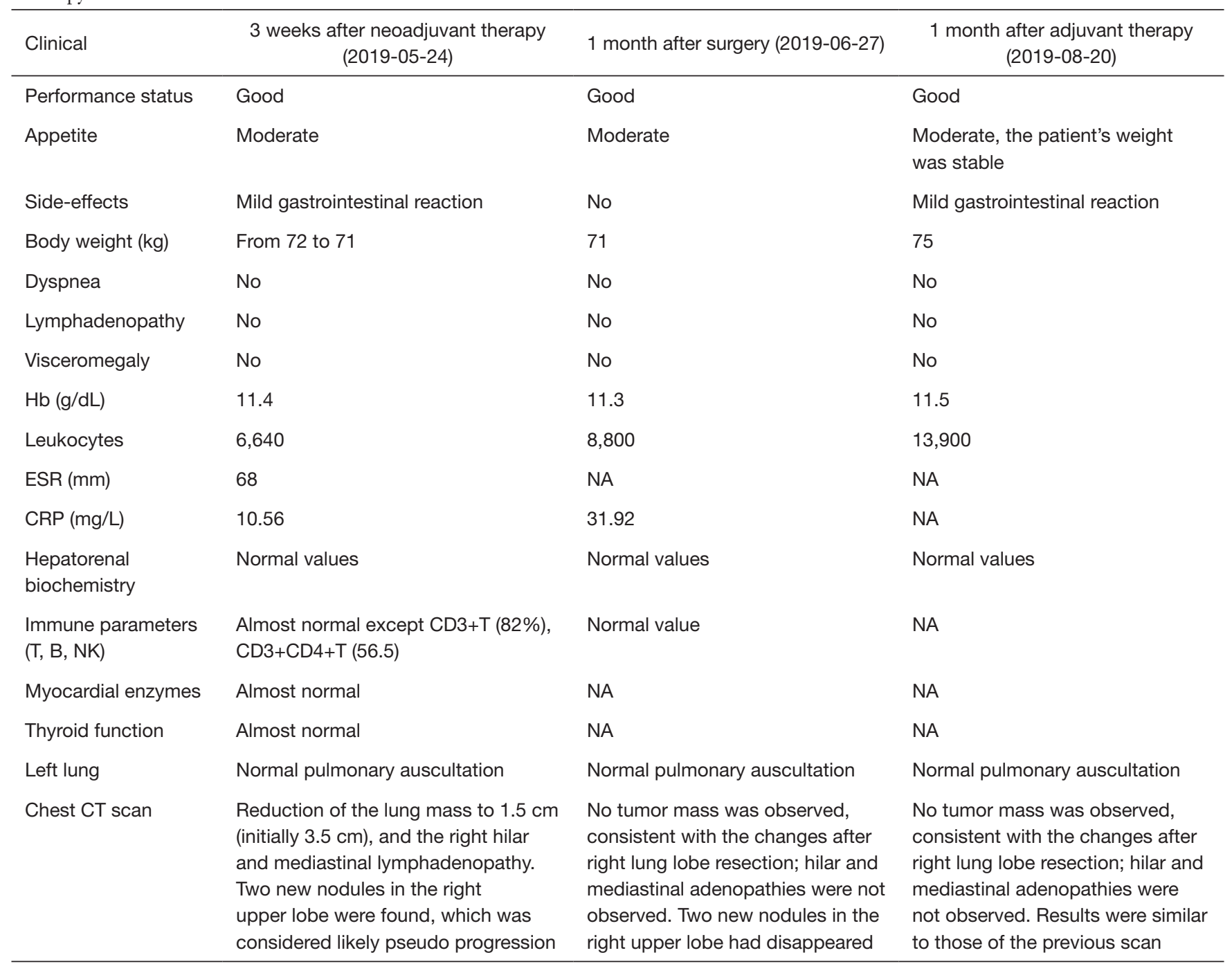

NA, not available.

rather than the growth of the tumor. Such phenomenon was also observed in a few other similar studies $(27,28)$.

Other preclinical tumor studies in mice suggested that PD-1 blockade could enhance the systemic activation of antitumor $\mathrm{T}$ cells, thereby potentially eliminating micrometastatic cancer, which may help to prevent tumor recurrence. It is also known that as tumors grow, they acquire mutations, some of which create neoantigens that influence the response of patients to ICIs. McGranahan et al. (29) revealed that CD8 (+) TILs reactive to clonal neoantigens were identified in early-stage NSCLC and expressed high levels of PD-1. PD-1 pathway blockade enhanced the activation of $\mathrm{T}$ cells in the early stages. The result of the NEOSTAR also showed that NI induced a higher percentage of non-viable tumor and of tissue resident memory TILs vs. nivolumab alone $(\mathrm{N})(\mathrm{CD} 3+$ $81.2 \%$ vs. $54.4 \%, \mathrm{P}=0.028 ; \mathrm{CD} 8+56.2 \%$ vs. $38.3 \%$, $\mathrm{P}=0.069)$. These data suggest that the blockade of $\mathrm{PD}-1$ pathway may enhance antitumor effects due to activation of host immunity and reduced tumor clonal heterogeneity.

\section{Can RECIST criteria also be applied to the evaluation neoadjuvant immunotherapy efficacy?}

It is widely acknowledged that RECIST-based radiographic evaluation in cancer immunotherapy trials may be 

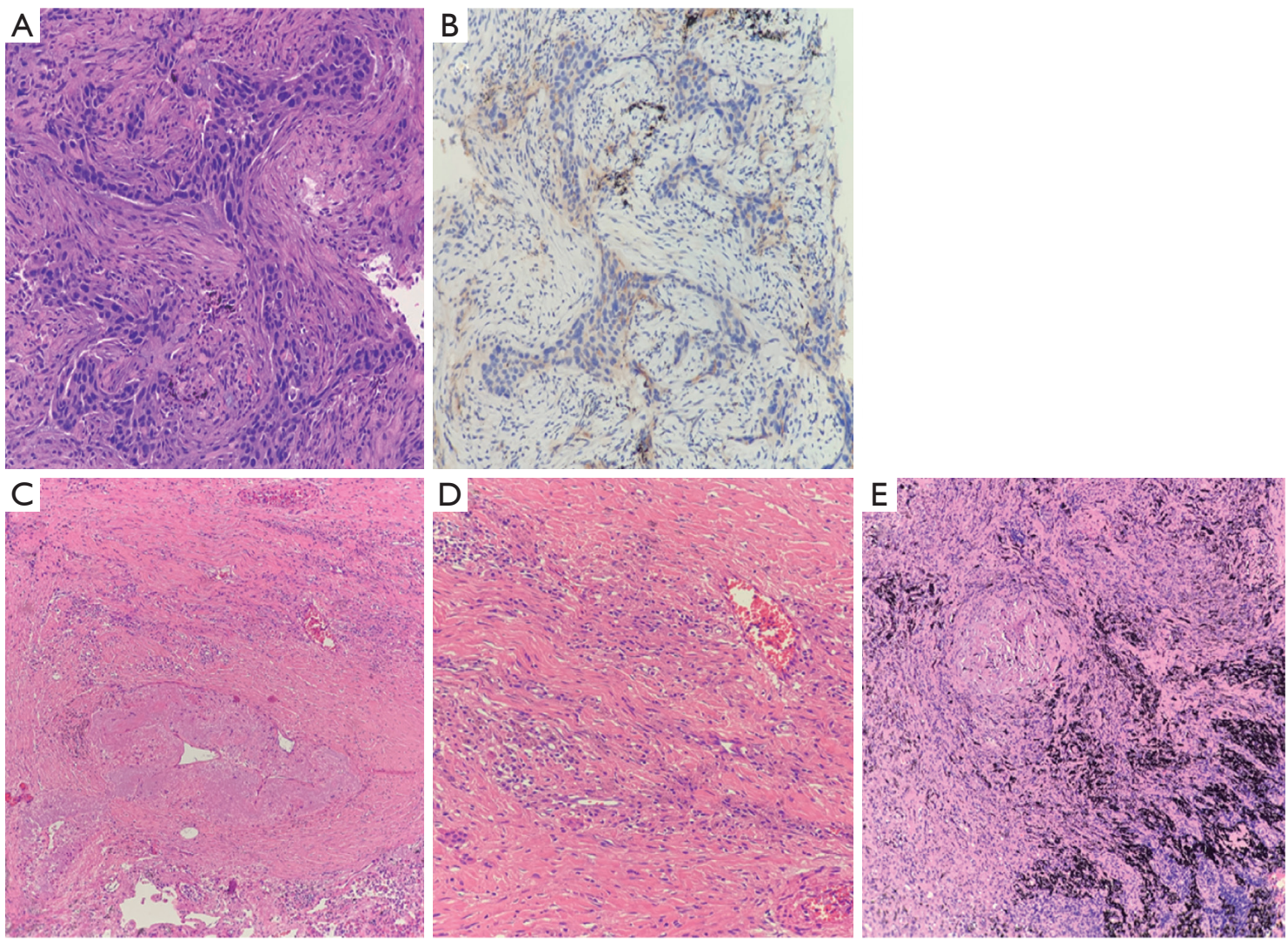

Figure 3 Histological characteristics of the lesions. Pre-neoadjuvant therapy hematoxylin-eosin staining for biopsy tissues of primary lung tumor (A) and immunochemistry results for PD-L1 expression (B) are shown (A,B, magnification $\times 20)$. Postsurgical intervention hematoxylin-eosin staining for the tumor bed $(\mathrm{C}$, magnification $\times 10$; D, magnification $\times 20)$ and mediastinal $4 \mathrm{R}$ lymphadenopathy $(\mathrm{E}$, magnification $\times 10$ ) are shown. Hyperplasia of the interstitial fibrous tissue around the dorsal bronchus of the lower lobe of the right lung with infiltration of lymphocytes was observed. No clear residues of the tumor were apparent. For the mediastinal 4R, the formation of fibrous nodules in lymph nodes with histocellular response was observed.

not be sufficiently accurate. The pCR of neoadjuvant immunotherapy is often not consistent with the imaging manifestations, and clinical manifestations of emerging immunoreactive nodules or pseudoprogression also tend to appear. Therefore, effectively predicting the clinical efficacy of neoadjuvant immunotherapy remains elusive.

iRECIST is a modified set of guidelines for response criteria in assessing immunotherapeutics, whose purpose is to ensure consistent design and trial data collection. The most prominent change in iRECIST is the introduction of immune unconfirmed progressive disease (iUPD) and immune confirmed progressive disease (iCPD) (30). Tazdait et al. analyzed response patterns and the discordance between RECIST 1.1, immune-related (ir) RECIST, and iRECIST guidelines, and explored the associations between response patterns and clinical outcome (31) (Table 3). The results showed that patients with atypical responses (pseudo progressions or dissociated response) had higher overall survival (OS) compared with those with true progression. Based on survival analyses, the RECIST 1.1 assessment underestimated the benefit of immunotherapy in $11 \%$ of the progressive patients. iRECIST and irRECIST identified these unconventional responses, with a 3.8\% discrepancy rate. Also, the application of PET-CT was helpful in evaluating the response to immunotherapy in NSCLC. Metabolic response, as an indicator of tumor activity, may provide more accurate information about objective response and disease progression. If patients with residual lesions can still be assessed by RECIST without fluorodeoxyglucose (FDG) uptake, they may achieve pCR. Harry et al. demonstrated that functional imaging can characterize tumor activity by measuring glucose uptake, perfusion, 
Table 3 Summary of iRECIST, irRECIST and RECIST Criteria

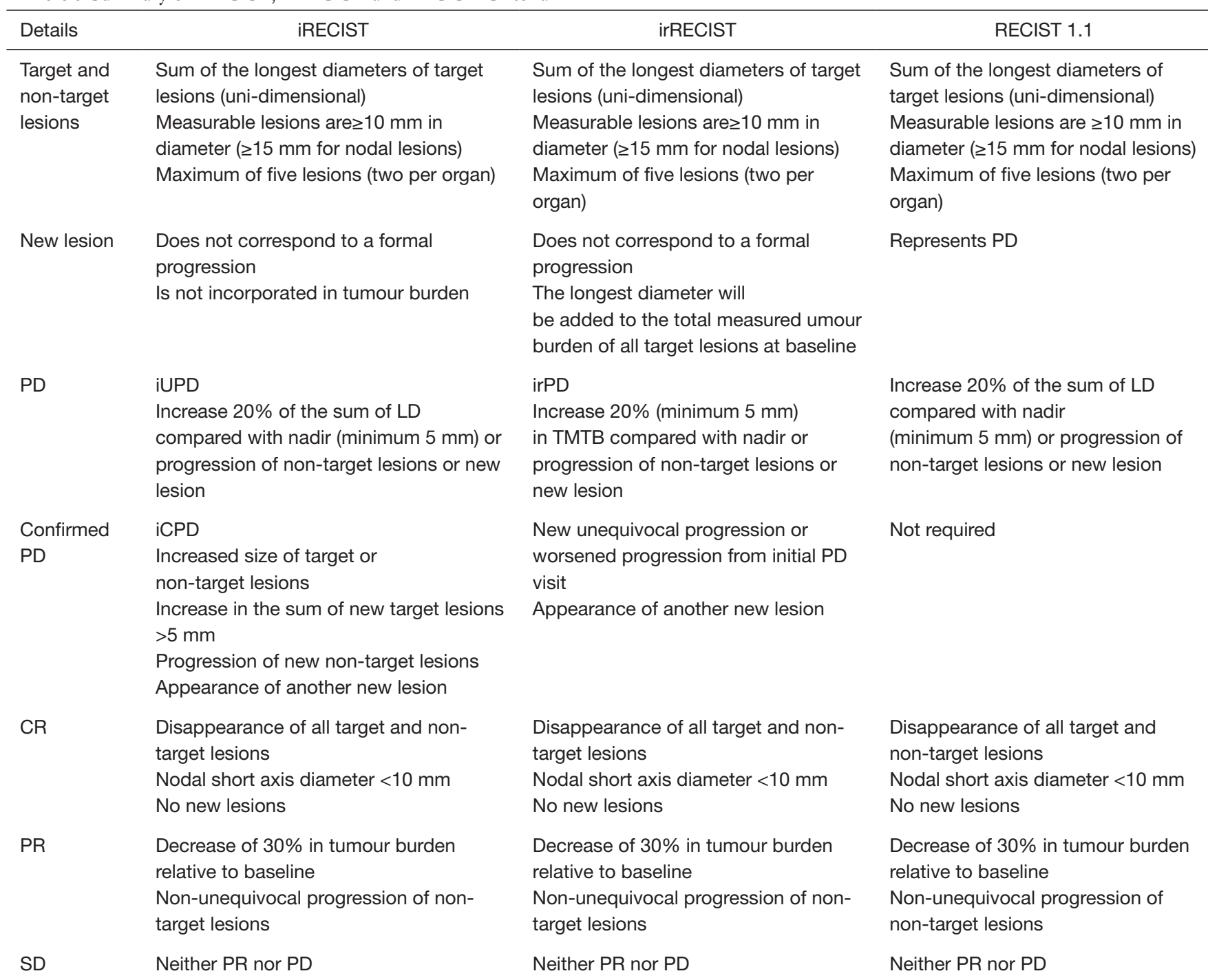

PD, progressive disease; iUPD, unconfirmed progressive disease; iCPD, confirmed progressive disease; LD, longest diameters; TMTB, total measured tumour burden. CR, complete response; PR, partial response; SD, stable disease,

hypoxia, and proliferation, all of which are potential indicators of tumor cell viability (32). 18F-FDG may also assist in the diagnosis of pseudo progression that increases in size but declines in FDG. Retrospective analyses have revealed that MPR after neoadjuvant chemotherapy was related to improved OS and disease-free survival (DFS). Chaft et al. examined neoadjuvant chemotherapy for nonsquamous NSCLC and reported that $22 \%$ of tumors showed MPR, with these responses being associated with long-term survival (18). Furthermore, MPR has been recommended as a surrogate endpoint $(33,34)$.

\section{What are the side effects and safety issues of neoadjuvant therapy? Do they affect the schedule of treatment completion?}

According to the results obtained in preliminary studies, neoadjuvant immunotherapy plus chemotherapy was well tolerated and rarely had side-effect-related delays in surgery. In the NADIM trial, 46 patients were recruited, 41 of whom had undergone surgery after neoadjuvant chemo-immunotherapy. Surgery was not delayed in any case. None of these patients withdrew from the study preoperatively due to progression or toxicity. In all, 41 
surgeries were performed, and all tumors were deemed resectable, with R0 resection in all patients. Additionally, the LCMC3 study showed that atezo in the neoadjuvant setting was well tolerated. There were two treatmentunrelated grade 5 adverse events (AEs) (cardiac death from postsurgical resection, death due to disease progression), 29 Gr 3-4 AEs [6 (6\%) treatment related], and 90 patients had surgery. Forde et al. reported similar results in patients with resectable lung cancer treated with neoadjuvant PD-1 blockade: neoadjuvant PD-1 blockade had an acceptable side-effect profile and was not associated with delays in surgery. Of the 21 tumors that were removed, 20 were completely resected (11). However, side-effects of neoadjuvant immunotherapy may still affect surgical treatment in resectable NSCLC. The NEOSTAR study reported that of the 44 patients who were randomly assigned, 3 patients received $<3$ doses due to treatmentrelated adverse events (TRAEs) (7\%), 34 patients had surgery after ICIs were administered, and 7 did not undergo resection. Surgical complications included 2 bronchopleural fistulas (BPFs) in $\mathrm{N}$ and 8 air leaks (5 N, $3 \mathrm{NI}$ ). Grade 3-5 TRAEs included a death due to BPF (grade $5, \mathrm{~N}$ ); grade 3 pneumonia, hypoxia, hypermagnesemia ( 1 each, all $\mathrm{N}$ ), and grade 3 diarrhea $(1 \mathrm{NI})$. As 5 patients failed to undergo surgery and 1 died perioperatively, neoadjuvant ICIs should thus be carefully selected for patients with resectable NSCLC.

\section{Is surgical intervention essential for patients with complete remission after neoadjuvant immunotherapy?}

There may exist scientific and clinical value in developing comprehensive therapy for solid tumors in NSCLC. In other solid tumors, including those of rectal cancer and esophageal cancer (35-39), vigorous surveillance without surgery may be considered if effective methods to predict pCR are available. Advances in chemoradiotherapy for patients with rectal cancer have delineated a select population of patients who can undergo pCR after surgery. In Brazil, surveillance of the patients who achieved pCR led to develop the "watch and wait" protocol (40). In this approach, patients are identified as having a clinical complete response (cCR) and followed with close surveillance by physical examination, endoscopic assessment, and imaging studies. Thus far, they have followed prospectively, a highly selected patient population, which had also been confirmed by other studies (41-43).

Results of studies in NSCLC suggest that pCR analysis may be helpful in confirming early signs of treatment efficacy years before survival data can be obtained, and may be able to determine the necessity for additional adjuvant therapy (44). There must be a balance struck between evaluation of $\mathrm{pCR}$ at an optimal timepoint to ensure it is a representative indicator of treatment-induced cell damage, without compromising surgical intervention and ultimate efficacy (45). Antonoff et al.'s univariate analysis (46) of preoperative chemoradiotherapy for superior sulcus NSCLC revealed that symptom improvement (alleviation of pain or neurological symptoms due to compression of the brachial plexus) and tumor remission by CT were correlated with pCR, rather than radiation dose. Further analysis showed that tumor size decline on radiographic evaluation before surgery may be an independent predictor of pCR (19). pCR rate in patients with both tumor remission and symptoms improvement was as high as $88 \%$.

However, from the basis of existing supportive surgery exemptions, it is still not feasible to do the following: (I) effectively predict pCR; (II) determine how long the immediate positive effect of immunotherapy will last, and whether secondary immune tolerance problems will occur; (III) ascertain whether tumor heterogeneity will contribute to the clinical value of local surgical intervention.

\section{Is it essential for patients who have been treated with neoadjuvant immunochemotherapy and surgical intervention to receive postoperative radiotherapy (PORT)?}

The role of PORT in the treatment of NSCLC patients with yp-TONOM0 remains clear. The main role of PORT is to reduce recurrence in NSCLC patients. Douillard et al. conducted a randomized study of adjuvant chemotherapy in the Adjuvant Navitas International Trial Association (ANITA) (47). An unplanned subset analysis of patients who received PORT was performed, and the results showed that PORT had a positive effect in patients with $\mathrm{pN} 2$ disease and had a negative effect in patients with pN1. Lally et al. (48) reported a retrospective study on a large database of NSCLC patients who had undergone PORT. Subset analysis revealed a significant improvement in survival for patients with $\mathrm{N} 2$ disease ( $\mathrm{HR}, 0.8555 ; \mathrm{P}=0.0077)$, but a decrease in survival in patients with $\mathrm{N} 0$ (heart rate, 1.1176; $\mathrm{P}=0.0435)$ and $\mathrm{N} 1$ disease (HR, 1.097; $\mathrm{P}=0.0196)$. Other similar studies also suggest that PORT may be appropriate for patients with high-risk of local recurrence, such as for patients with $\mathrm{cN} 2$ disease and multiple mediastinal lymph node metastasis by post-operative pathology (49). 
Kwiatkowski et al. performed an interim analysis of biomarker data from a multicenter study (LCMC3) in resectable NSCLC patients who received neoadjuvant atezo (50). The results showed that 4 of 82 patients had pCR (pCR: 5\%), 6 (7.3\%) had PR, 72 (87.8\%) had SD, and $4(4.9 \%)$ had PD. With a 12 -month DFS rate of $89 \%$, only 6 patients experienced postoperative recurrence. Thus, PORT in this population of NSCLC patient might be not essential. Further prospective studies are needed to confirm the role of PORT in yp-T0N0M0 NSCLC patients who receive neoadjuvant and adjuvant $\mathrm{PD}-1$ blockade and chemotherapy.

\section{Can concurrent radiotherapy chemotherapy plus immunotherapy replace surgery?}

In the era of chemotherapeutic agents, the current clinical trial evidence suggests that there is no significant difference between surgery and concurrent chemoradiotherapy after neoadjuvant therapy for stage III NSCLC (51). Eberhardt et al. reported OS rates of $44 \%$ for surgery and $40 \%$ for chemoradiotherapy, and PFS rates of $32 \%$ for surgery and $35 \%$ for chemoradiotherapy. With a median follow-up of 78 months, 5-year OS and PFS did not differ significantly between these two different treatments. Based on the phase 3 PACIFIC trial $(9,52,53)$ study of patients with unresectable stage III NSCLC without progression after chemoradiotherapy, Gray et al. (54) updated the OS data from the PACIFIC trial in 2019 and found that durvalumab did not reach median OS, but the median OS with placebo was 29.1 months. The 12-, 24- and 36-month OS rates with durvalumab and placebo were $83.1 \%$ vs. $74.6 \%, 66.3 \%$ vs. $55.3 \%$, and $57.0 \%$ vs. $43.5 \%$, respectively. The PACIFIC study indicated a long-term clinical benefit could be gained with immunotherapy after chemoradiotherapy, which is established as the standard treatment in unresectable stage III NSCLC; however, further research as to whether there is a difference between neoadjuvant therapy surgery and concurrent chemoradiotherapy followed by immunotherapy is needed. There is a possibility that concurrent chemoradiotherapy plus consolidation immunotherapy may replace surgery in the future.

\section{Which is the optimal comprehensive therapeutic mode to combine with ICIs for stage III NSCLC?}

Preliminary studies of pembrolizumab have also shown encouraging results, and multiple studies are ongoing, including two phase III studies: PEARLS and KEYNOTE-671. PEARLS (MK-3475-091/ KEYNOTE-091) was initiated to study pembrolizumab (mk-3475) vs. placebo for participants with NSCLC after resection with or without standard adjuvant therapy. Meanwhile, Fernando et al. (55) conducted a randomized, double-blind phase 3 study (KEYNOTE-671) to evaluate neoadjuvant platinum-based chemotherapy with perioperative pembrolizumab or placebo in resectable stage IIB or IIIA NSCLC. Other therapeutic modes that combine with ICIs for patients with stage III unresectable NSCLC also exist (Table 4).

The PACIFIC trial was the first to certify the efficacy of durvalumab, and it provoked several further questions. The first concern is the timing of initiation of consolidation therapy. Uemura et al. (52) demonstrated a better PFS when durvalumab was given within $\leq 2$ weeks of radiotherapy (HR, 0.39; 95\% CI, 0.26-058), as opposed to >2 weeks postradiation (HR, 0.63 ; 95\% CI, 0.49-0.80). It appears then that less than 2 weeks may be a more appropriate time. However, the mode of previous treatment should also be taken into account for the timing of initiation adjuvant immunotherapy.

The second issue is how long adjuvant immunotherapy should last. At present, there is no definitive evidence to resolve this issue, as the relevant clinical studies have offered a variety of conclusions. Patients in the PACIFIC trial received durvalumab or placebo, $10 \mathrm{mg} / \mathrm{kg}$ intravenously every 2 weeks, for up to 12 months, which demonstrated long-term clinical benefit with adjuvant immunotherapy after chemoradiotherapy. A phase 3 clinical trial (NCT03728556) in Guangzhou is recruiting patients with NSCLC to evaluate the safety and efficacy of CS1001. An estimated 402 participants with stage III disease will be randomized to receive either CS1001 or a placebo, every 3 weeks for up to 2 years. In the study of cisplatin and etoposide plus radiation followed by nivolumab/placebo for locally advanced NSCLC (NCT02768558), patients with stage III unresectable disease will receive thoracic radiation, cisplatin, and etoposide followed by nivolumab or placebo given every 2 weeks for a year. In the study of consolidation sintilimab after concurrent chemoradiation in patients with unresectable stage III NSCLC (consist), $200 \mathrm{mg}$ of sintilimab will be administered intravenously after concurrent chemoradiation, every 3 weeks until PD. In a phase 1 clinical trial (NCT02987998) for NSCLC, consolidation pembrolizumab will be given following the initial treatment (200 mg IV D1, 21, 42). Furthermore, 
Table 4 Therapeutic modes for stage III unresectable non-small cell lung cancer (NSCLC)

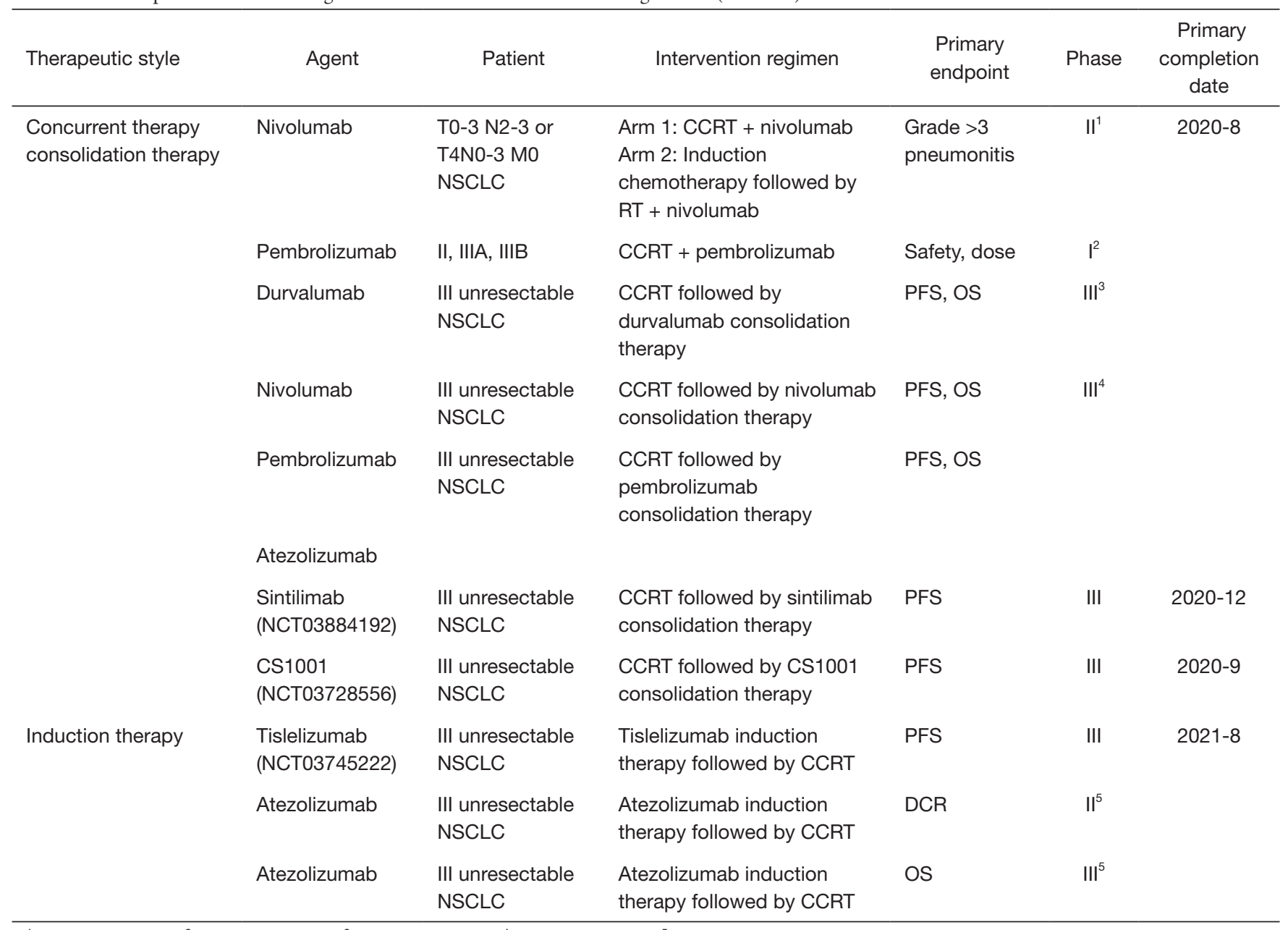

1, NCT02434081; ${ }^{2}$, NCT02621398; ${ }^{3}$, NCT02125461; ${ }^{4}$, NCT02768558; ${ }^{5}$, NCT03102242.

a new randomized, placebo-controlled, phase 3 trial (PACIFIC-5) was conducted in China, which evaluated durvalumab in patients with stage III, unresectable NSCLC who did not have disease progression after chemoradiotherapy. Patients were randomized, in a 2:1 ratio, to receive durvalumab intravenously, at a dose of $1,500 \mathrm{mg}$, or to receive a matching placebo every 4 weeks, until disease progression. Randomization occurred 1 to 28 days after chemoradiotherapy and was stratified according to staging of disease and previous treatment. The primary end points were PFS and OS. The results of the trial are expected to be revealed shortly.

The third problem related to identifying the most comprehensive treatment strategy. With the inclusion of surgery, it is necessary to arrange the time sequence and intensity of ICIs, radiation, and chemotherapy.
Meanwhile, it is also important to determine whether there is a difference between non-surgery and surgery, or how much benefits can surgery bring, especially for patients who achieved CR after neoadjuvant therapy. Thus, further prospective studies are indispensable to confirm the best treatment strategy.

\section{Personalized treatment for stage III NSCLC}

Personalized treatment will inevitably become the focus of future research. In order to choose the appropriate comprehensive treatment strategy, big data analytics, which is based on basic clinical data, biomics data, and radiomics data, may become an integral part of therapy. Using big data, valuable information will be extracted from patients with different clinical biological behaviors and failure 
phenotypes, which could be used as a guide for precision therapy. Rizvi et al. analyzed molecular determinants of responses to PD-1 and PD-L1 blockade in patients with NSCLC profiled with targeted next-generation sequencing, and the results demonstrated that targeted NGS could accurately estimate TMB and that elevated TMB further improved the likelihood of benefit to ICIs. TMB did not correlate with PD-L1 expression, with both variables having similar predictive power (56). Similar results are reported in advanced NSCLC and in most other cancers (57-60). Currently, other indicators from the microenvironment $(61,62)$, include CD8, CD45RO, CD3+, S100 + cells etc. However, the treatment strategy for patients with stage III NSCLC is so complex that prediction is exceedingly difficult, and requires more reliable, comprehensive, and dynamic predictive measures.

In short, immunotherapy has opened an expansive exploratory space to discover further improvements in the clinical efficacy of patients with stage III NSCLC, but has, at the same time, posed numerous challenges. More clinical studies and further exploration of personalized treatment, guided by clinical, pathological, biomarkers, and imaging data are needed to help these patients.

\section{Acknowledgments}

Funding: This study was supported by the National Key Research and Development Program of China (2016YFC0905502), the Shanghai Shen Kang Hospital Development Center Clinical Research Plan of SHDC (16CR1016A), and the National Natural Science Foundation of China (81803044).

\section{Footnote}

Conflicts of Interest: All authors have completed the ICMJE uniform disclosure form (available at http://dx.doi. org/10.21037/tlcr-20-896). CZ serves as an unpaid editorin-chief of Translational Lung Cancer Research. YH serves as the unpaid editorial board member of Translational Lung Cancer Research from January 2020 to December 2021. The other authors have no conflicts of interest to declare.

Ethical Statement: The authors are accountable for all aspects of the work in ensuring that questions related to the accuracy or integrity of any part of the work are appropriately investigated and resolved. The study was conducted in accordance with the Declaration of Helsinki (as revised in 2013). Written informed consent was obtained from the patient for publication of this manuscript and any accompanying images.

Open Access Statement: This is an Open Access article distributed in accordance with the Creative Commons Attribution-NonCommercial-NoDerivs 4.0 International License (CC BY-NC-ND 4.0), which permits the noncommercial replication and distribution of the article with the strict proviso that no changes or edits are made and the original work is properly cited (including links to both the formal publication through the relevant DOI and the license). See: https://creativecommons.org/licenses/by-nc-nd/4.0/.

\section{References}

1. Malapelle U, Iaccarino A, Rossi A. Cytology versus histology for programmed death-ligand 1 expression evaluation in the landscape of non-small cell lung cancer patients selection for immunotherapy. Transl Lung Cancer Res 2018;7:S221-4.

2. Morgensztern D, Ng SH, Gao F, et al. Trends in Stage Distribution for Patients with Non-small Cell Lung Cancer: A National Cancer Database Survey. J Thorac Oncol 2010;5:29-33.

3. Pignon JP, Tribodet H, Scagliotti GV, et al. Lung Adjuvant Cisplatin Evaluation: A Pooled Analysis by the LACE Collaborative Group. J Clin Oncol 2008;26:3552-9.

4. Wakelee HA, Dahlberg SE, Keller SM, et al. Adjuvant chemotherapy with or without bevacizumab in patients with resected non-small-cell lung cancer (E1505): an openlabel, multicentre, randomised, phase 3 trial. Lancet Oncol 2017;18:1610-23.

5. Hodkinson PS, Mackinnon A, Sethi T. Targeting growth factors in lung cancer. Chest 2008;133:1209-16.

6. Gettinger SN, Horn L, Gandhi L, et al. Overall Survival and Long-Term Safety of Nivolumab (Anti-Programmed Death 1 Antibody. J Clin Oncol 2015;33:2004-12.

7. Rittmeyer A, Barlesi F, Waterkamp D, et al. Atezolizumab versus docetaxel in patients with previously treated non-small-cell lung cancer (OAK): a phase 3, openlabel, multicentre randomised controlled trial. Lancet 2017;389:255-65.

8. Reck M, Rodríguez-Abreu D, Robinson AG, et al. Pembrolizumab versus Chemotherapy for PD-L1Positive Non-Small-Cell Lung Cancer. N Engl J Med 2016;375:1823-33.

9. Tsiara A, Liontos M, Kaparelou M, et al. Implementation 
of immunotherapy in the treatment of advanced non-small cell lung cancer (NSCLC). Ann Transl Med 2018;6:144.

10. Garon EB, Rizvi NA, Hui R, et al. Pembrolizumab for the Treatment of Non-Small-Cell Lung Cancer. N Engl J Med 2015;372;2018-28.

11. Forde PM, Chaft JE, Smith KN, et al. Neoadjuvant PD-1 Blockade in Resectable Lung Cancer. N Engl J Med 2018;378:1976-86.

12. Riaz R, Lande L, Zappetti D. Pembrolizumab Plus Chemotherapy in Patients With Previously Untreated Metastatic Non-small Cell Lung Cancer Without EGFR or ALK Mutations is Superior to Chemotherapy Alone. Clinical Pulmonary Medicine 2018;25:194-5.

13. Mok TSK, Wu YL, Kudaba I, et al. Pembrolizumab versus chemotherapy for previously untreated, PD-L1expressing, locally advanced or metastatic non-small-cell lung cancer (KEYNOTE-042): a randomised, open-label, controlled, phase 3 trial. Lancet 2019;393:1819-30.

14. Herbst RS, Baas P, Kim DW, et al. Pembrolizumab versus docetaxel for previously treated, PD-L1-positive, advanced non-small-cell lung cancer (KEYNOTE-010): a randomised controlled trial. Lancet 2016;387:1540-50.

15. Gandhi L, Rodríguez-Abreu D, Gadgeel S, et al. Pembrolizumab plus Chemotherapy in Metastatic NonSmall-Cell Lung Cancer. N Engl J Med 2018;378:2078-92.

16. Sawrie SM, Guthrie BL, Spencer SA, et al. Predictors of distant brain recurrence for patients with newly diagnosed brain metastases treated with stereotactic radiosurgery alone. Int J Radiat Oncol Biol Phys 2008;70:181-6.

17. Kocher F, Pircher A, Mohn-Staudner A, et al. Multicenter phase II study evaluating docetaxel and cisplatin as neoadjuvant induction regimen prior to surgery or radiochemotherapy with docetaxel, followed by adjuvant docetaxel therapy in chemonaive patients with NSCLC stage II, IIIA and IIIB (TAX-AT 1. Lung Cancer 2014;85:395-400.

18. Chaft JE, Rusch V, Ginsberg MS, et al. Phase II Trial of Neoadjuvant Bevacizumab Plus Chemotherapy and Adjuvant Bevacizumab in Patients with Resectable Nonsquamous Non-Small-Cell Lung Cancers. J Thorac Oncol 2013;8:1084-90.

19. Antonoff MB, Hofstetter WL, Correa AM, et al. Clinical Prediction of Pathologic Complete Response in Superior Sulcus Non-Small Cell Lung Cancer. Ann Thorac Surg 2016;101:211-7.

20. Haque W, Verma V, Butler EB, et al. Pathologic nodal clearance and complete response following neoadjuvant chemoradiation for clinical N2 non-small cell lung cancer: Predictors and long-term outcomes. Lung Cancer 2019;130:93-100.

21. Albain KS, Swann RS, Rusch VW, et al. Radiotherapy plus chemotherapy with or without surgical resection for stage III non-small-cell lung cancer: A phase III randomised controlled trial. Lancet 2009;374:379-86.

22. Kernstine KH, Moon J, Kraut MJ, et al. Trimodality Therapy for Superior Sulcus Non-Small Cell Lung Cancer: Southwest Oncology Group-Intergroup Trial S0220. Ann Thorac Surg 2014;98:402-10.

23. Tsuboi M, Kunitoh H, Shibata T, et al. Seven-year followup of preoperative chemoradiotherapy in superior sulcus tumor: Report of a Japan Clinical Oncology Group Trial (JCOG9806). J Clin Oncol 2010;28:abstr 7025.

24. Kunitoh H, Kato H, Tsuboi M, et al. Phase II Trial of Preoperative Chemoradiotherapy Followed by Surgical Resection in Patients With Superior Sulcus Non-SmallCell Lung Cancers: Report of Japan Clinical Oncology Group Trial 9806. J Clin Oncol 2008;26:644-9.

25. Rusch VW, Giroux DJ, Kraut MJ, et al. Induction Chemoradiation and Surgical Resection for Superior Sulcus Non-Small-Cell Lung Carcinomas: LongTerm Results of Southwest Oncology Group Trial 9416 (Intergroup Trial 0160). J Clin Oncol 2007;25:313-8.

26. Rusch VW, Giroux DJ, Kraut MJ, et al. Induction chemoradiation and surgical resection for non-small cell lung carcinomas of the superior sulcus: Initial results of Southwest Oncology Group Trial 9416 (Intergroup Trial 0160). J Thorac Cardiovasc Surg 2001;121:472-83.

27. Ribas A, Chmielowski B, Glaspy JA. Do We Need a Different Set of Response Assessment Criteria for Tumor Immunotherapy? Clin Cancer Res 2009;15:7116-8.

28. Hodi FS, Hwu WJ, Kefford R, et al. Evaluation of Immune-Related Response Criteria and RECIST v1.1 in Patients With Advanced Melanoma Treated With Pembrolizumab. J Clin Oncol 2016;34:1510-7.

29. McGranahan N, Furness AJ, Rosenthal R, et al. Clonal neoantigens elicit $\mathrm{T}$ cell immunoreactivity and sensitivity to immune checkpoint blockade. Science 2016;351:1463-9.

30. Seymour L, Bogaerts J, Perrone A, et al. iRECIST: guidelines for response criteria for use in trials testing immunotherapeutics. Lancet Oncol 2017;18:e143-52.

31. Tazdait M, Mezquita L, Lahmar J, et al. Patterns of responses in metastatic NSCLC during PD-1 or PDL-1 inhibitor therapy: Comparison of RECIST 1.1, irRECIST and iRECIST criteria. Eur J Cancer 2018;88:38-47.

32. Harry VN, Semple SI, Parkin DE, et al. Use of new imaging techniques to predict tumour response to therapy. 
Lancet Oncol 2010;11:92-102.

33. Hellmann MD, Chaft JE, William WN, et al. Pathological response after neoadjuvant chemotherapy in resectable non-small-cell lung cancers: proposal for the use of major pathological response as a surrogate endpoint. Lancet Oncol 2014;15:e42-50.

34. Pataer A, Kalhor N, Correa AM, et al. Histopathologic Response Criteria Predict Survival of Patients with Resected Lung Cancer After Neoadjuvant Chemotherapy. J Thorac Oncol 2012;7:825-32.

35. Ghia A, Tome WA, Thomas S, et al. Distribution of brain metastases in relation to the hippocampus: implications for neurocognitive functional preservation. Int J Radiat Oncol Biol Phys 2007;68:971-7.

36. Gondi V, Tome WA, Marsh J, et al. Estimated risk of perihippocampal disease progression after hippocampal avoidance during whole-brain radiotherapy: safety profile for RTOG 0933. Radiother Oncol 2010;95:327-31.

37. Chao YK, Tseng CK, Wen YW, et al. Using Pretreatment Tumor Depth and Length to Select Esophageal Squamous Cell Carcinoma Patients for Nonoperative Treatment After Neoadjuvant Chemoradiotherapy. Ann Surg Oncol 2013;20:3000-8.

38. Jeonq YR, Kim JH, Kim SB, et al. 6519 POSTER Role of Surgical Resection in Complete Responders on FDGPET After Chemoradiotherapy for the Locally Advanced Esophageal Cancer. Eur J Cancer 2011;47:S447-8.

39. Cavallin F, Scarpa M, Alfieri R, et al. The Role of Surgery in Patients With a Complete Clinical Response After Chemoradiation for Esophageal Cancer. Ann Surg 2015;262:e101.

40. Habr-Gama A, Sabbaga J, Gama-Rodrigues J, et al. Watch and Wait Approach Following Extended Neoadjuvant Chemoradiation for Distal Rectal Cancer: Are We Getting Closer to Anal Cancer Management? Dis Colon Rectum 2013;56:1109-17.

41. Maas M, Beetstan RG, Lambregts DM, et al. Waitand-see policy for clinical complete responders after chemoradiation for rectal cancer. J Clin Oncol 2011;29:4633-40.

42. Smith JD, Ruby JA, Goodman KA, et al. Nonoperative management of rectal cancer with complete clinical response after neoadjuvant therapy. Ann Surg 2012;256:965-72.

43. Pozo ME, Fang SH. Watch and wait approach to rectal cancer: A review. World J Gastrointest Surg 2015;7:306-12.

44. El Kadmiri MAA, Rajan A. Neoadjuvant immunotherapy for non-small cell lung cancer: can early intervention result in durable clinical benefit? J Thorac Dis 2018;10:S3203-6.

45. Samson P, Crabtree TD, Robinson CG, et al. Defining the Ideal Time Interval Between Planned Induction Therapy and Surgery for Stage IIIA Non-Small Cell Lung Cancer. Ann Thorac Surg 2017;103:1070-5.

46. Antonoff MB, Hofstetter WL, Correa AM, et al. Clinical Prediction of Pathologic Complete Response in Superior Sulcus Non-Small Cell LungCancer. Ann Thorac Surg 2016;101:211-7.

47. Douillard JY, Rosell R, Lena MD, et al. Adjuvant vinorelbine plus cisplatin versus observation in patients with completely resected stage IB-IIIA non-small-cell lung cancer (Adjuvant Navelbine International Trialist Association [ANITA]): a randomised controlled trial. Lancet Oncol 2006;7:719-27.

48. Lally BE, Zelterman D, Colasanto JM, et al. Postoperative radiotherapy for stage II or III non-small-cell lung cancer using the surveillance, epidemiology, and end results database. J Clin Oncol 2006;24:2998-3006.

49. Robinson CG, Patel AP, Bradley JD, et al. Postoperative Radiotherapy for Pathologic N2 Non-Small-Cell Lung Cancer Treated With Adjuvant Chemotherapy: A Review of the National Cancer Data Base. J Clin Oncol 2015;33:870-6.

50. Kwiatkowski DJ, Rusch VW, Chaft JE, et al. Neoadjuvant atezolizumab in resectable non-small cell lung cancer (NSCLC): Interim analysis and biomarker data from a multicenter study (LCMC3). J Clin Oncol 2019;37:abstr 8503.

51. Eberhardt WE, Pöttgen C, Gauler TC, et al. Phase III Study of Surgery Versus Definitive Concurrent Chemoradiotherapy Boost in Patients With Resectable Stage IIIA(N2) and Selected IIIB Non-Small-Cell Lung Cancer After Induction Chemotherapy and Concurrent Chemoradiotherapy (ESPATUE). J Clin Oncol 2015;33:4194-201.

52. Uemura T, Hida T. Durvalumab showed long and durable effects after chemoradiotherapy in stage III non-small cell lung cancer: results of the PACIFIC study. J Thorac Dis 2018;10:S1108-12.

53. Antonia SJ, Villegas A, Daniel D, et al. Overall Survival with Durvalumab after Chemoradiotherapy in Stage III NSCLC. N Engl J Med 2018;379:2342-50.

54. Gray JE, Villegas A, Daniel D, et al. Three-Year Overall Survival with Durvalumab after Chemoradiotherapy in Stage III NSCLC-Update from PACIFIC. J Thorac Oncol 2020;15:288-93.

55. Fernando HC, Yang J, Ferraro GL, et al. Randomized, 
double-blind phase 3 study evaluating neoadjuvant platinum-based chemotherapy with perioperative pembrolizumab or placebo in resectable stage IIB or IIIA NSCLC: KEYNOTE-671. J Clin Oncol 2018;36:abstr TPS8583.

56. Rizvi H, Sanchez-Vega F, Chatila W, et al. Molecular Determinants of Response to Anti-Programmed Cell Death (PD)-1 and Anti-Programmed Death-Ligand (PDL)-Ligand 1 Blockade in Patients With Non-Small-Cell Lung Cancer Profiled With Targeted Next-Generation Sequencing. J Clin Oncol 2018;36:633-41.

57. Hellmann MD, Nathanson T, Rizvi H, et al. Genomic Features of Response to Combination Immunotherapy in Patients with Advanced Non-Small-Cell Lung Cancer. Cancer Cell 2018;33:843-52.e4.

58. Olmez I, Donahue BR, Butler JS, et al. Clinical outcomes in extracranial tumor sites and unusual toxicities with concurrent whole brain radiation (WBRT) and Erlotinib treatment in patients with non-small cell lung

Cite this article as: Yang ZR, Liu MN, Yu JH, Yang YH, Chen TX, Han YC, Zhu L, Zhao JK, Fu XL, Cai XW. Treatment of stage III non-small cell lung cancer in the era of immunotherapy: pathological complete response to neoadjuvant pembrolizumab and chemotherapy. Transl Lung Cancer Res 2020;9(5):2059-2073. doi: 10.21037/tlcr-20-896 cancer (NSCLC) with brain metastasis. Lung Cancer 2010;70:174-9.

59. Dempke WCM, Fenchel K, Dale SP. Programmed cell death ligand-1 (PD-L1) as a biomarker for non-small cell lung cancer (NSCLC) treatment-are we barking up the wrong tree? Transl Lung Cancer Res 2018;7:S275-9.

60. Yarchoan M, Hopkins A, Jaffee EM. Tumor Mutational Burden and Response Rate to PD-1 Inhibition. N Engl J Med 2017;377:2500-1.

61. Yoneda K, Kuwata T, Kanayama M, et al. Alteration in tumoural PD-L1 expression and stromal CD8-positive tumour-infiltrating lymphocytes after concurrent chemoradiotherapy for non-small cell lung cancer. Br J Cancer 2019;121:490-6.

62. Johnson SK, Kerr KM, Chapman AD, et al. Immune cell infiltrates and prognosis in primary carcinoma of the lung. Lung Cancer 2000;27:27-35.

(English Language Editor: J. Gray) 